\title{
Carbon C 13 Lycopene
}

National Cancer Institute

\section{Source}

National Cancer Institute. Carbon C 13 Lycopene. NCI Thesaurus. Code C99120.

Carbon C 13 labeled lycopene used as a tracer for carotenoid metabolism studies in vivo. After oral administration of carbon C 13 lycopene, in combination with C 13 labeled phytoene and phytofluene, the absorption kinetics, distribution patterns, metabolism and targets of these carotenoids can be measured upon imaging of the non-radioactive C 13 . Lycopene, a carotenoid pigment found in high concentrations in tomatoes as well as in other fruits and vegetables, serves as an antioxidant in vivo; its intake has been associated with a reduced risk of certain types of cancer and cardiovascular diseases. 